\title{
Predictors of Nodal and Metastatic Failure in Early Stage Non-Small Cell Lung Cancer after Stereotactic Body Radiation Therapy
}

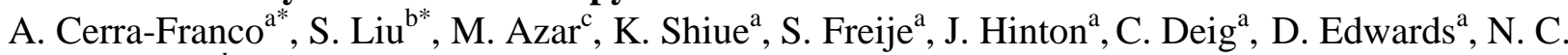
Estabrook III $^{\text {d }}$, S. G. Ellsworth ${ }^{\mathrm{a}}$, K. Huang ${ }^{\mathrm{a}}$, K. Diab ${ }^{\mathrm{e}}$, M. P. Langer ${ }^{\mathrm{a}}$, R. Zellars ${ }^{\mathrm{a}}$, F. M. Kong ${ }^{\mathrm{a}}$, J.

$\mathrm{Wan}^{\mathrm{b}}$, and T. Lautenschlaeger ${ }^{\mathrm{a}}$

*equal contribution

${ }^{\mathrm{a}}$ Department of Radiation Oncology

Simon Cancer Center

Indiana University School of Medicine

535 Barnhill Dr, RT 041

Indianapolis, IN 46202

${ }^{\mathrm{b}}$ Department of Medical and Molecular Genetics

Collaborative Core for Cancer Bioinformatics

410 W. 10th Street, HITS Building 4000

Indianapolis, IN 46202

${ }^{c}$ Department of Internal Medicine

Indiana University School of Medicine

1120 W. Michigan Street

Gatch Hall, CL 630

Indianapolis, IN 46202

${ }^{\mathrm{d}}$ Department of Radiation Oncology

Indiana University Health Arnett Hospital

420 N 26th St

Lafayette, IN 47904

${ }^{\mathrm{e}}$ Pulmonary, Critical Care, Sleep \& Occupational Medicine

Indiana University School of Medicine

Gatch Hall, CL260

1120 W. Michigan Street

Indianapolis, IN 46202

Corresponding author:

Tim Lautenschlaeger, MD

Department of Radiation Oncology

Simon Cancer Center

Indiana University School of Medicine

535 Barnhill Dr, RT 041

Indianapolis, IN 46202

E-mail: timlaut@iupui.edu

Phone: 317.278.7237

This is the author's manuscript of the article published in final edited form as:

Cerra-Franco, A., Liu, S., Azar, M., Shiue, K., Freije, S., Hinton, J., ... Lautenschlaeger, T. (2018). Predictors of Nodal and Metastatic Failure in Early Stage Non-Small Cell Lung Cancer after Stereotactic Body Radiation Therapy. Clinical Lung Cancer. https://doi.org/10.1016/j.cllc.2018.12.016 
Conflicts of Interest:

No author report any conflicts of interest. 


\section{MicroAbstract:}

Many ES-NSCLC patients develop metastases after SBRT. We retrospectively analyzed multiple variables in 406 lesions to develop a predictor for metastatic failure. GTV volume and prescription dose were significantly associated with metastases. A metastasis risk score tool was developed to identify patients at higher risk for metastases after lung SBRT who might benefit from enrollment in future trials evaluating the benefit of adjuvant or intensified treatment.

\section{Abstract}

\section{Introduction/Background:}

Many early-stage non-small cell lung cancer (ES-NSCLC) patients undergoing stereotactic body radiation therapy (SBRT) develop metastases, which is associated with poor outcomes. We sought to identify factors predictive of metastases after lung SBRT and created a risk stratification tool.

\section{Materials and Methods:}

We included 363 patients with ES-NSCLC who received SBRT; median follow-up was 5.8 years. The following patient and tumor factors were retrospectively analyzed for their association with metastases (defined as nodal and/or distant failure): sex; age; lobe involved; centrality; previous NSCLC; smoking status; gross tumor volume (GTV); T-stage; histology; dose; minimum, maximum, and mean GTV dose; and parenchymal lung failure. A metastasis risk-score linearmodel using beta coefficients from a multivariate Cox model was built.

\section{Results:}

A total of 111/406 (27.3\%) lesions metastasized. GTV volume and dose were significantly associated with metastases on univariate and multivariate Cox proportional hazards modeling ( $p<0.001$ and $\mathrm{HR}=1.02$ per $\mathrm{mL}, \mathrm{p}<0.05$ and $\mathrm{HR}=0.99$ per $\mathrm{Gy}$, respectively). Histology, $\mathrm{T}$-stage, centrality, lung parenchymal failures, and previous NSCLC were not associated with development of metastasis. A metastasis risk-score model using GTV volume and prescription dose was built: [risk score $=(0.01611 \times$ GTV $)-\left(0.00525 \times\right.$ dose $\left.\left.\left(\mathrm{BED}_{10}\right)\right)\right]$. Two risk-score cutoffs separating the cohort into low-, medium-, and high-risk subgroups were examined. The risk-score identified significant differences in time to metastases between low-, medium-, and high-risk patients $(\mathrm{p}<0.001)$, with 3 -year estimates of $81.1 \%, 63.8 \%$, and $38 \%$, respectively.

\section{Conclusion:}

GTV volume and radiation dose are associated with time to metastasis and may be used to identify patients at higher risk of metastasis after lung SBRT.

Key words: SBRT, SABR, radiation, stereotactic body ablative radiotherapy, metastasis 


\section{Introduction}

The current standard of care for early-stage non-small cell lung cancer (NSCLC) without lymph node involvement is tumor resection with hilar and mediastinal node dissection. For patients who are medically inoperable or refuse surgery, stereotactic body radiation therapy (SBRT), also known as stereotactic ablative radiotherapy, is the standard of care. Multiple reports have shown excellent rates of local control after SBRT for early-stage NSCLC [1-6]. The Radiation Therapy Oncology Group conducted a phase II trial (RTOG 0236) evaluating the efficacy of SBRT for patients with peripherally located tumors. The reported 3-year local control (LC) rate was $98 \%$ with a 3-year overall survival (OS) of $56 \%$ and a median OS of 4 years [1]. A pooled analysis of two prematurely closed randomized trials comparing SBRT vs. lobectomy for operable stage I (T1-2aN0M0) NSCLC patients suggested at least clinical equipoise between SBRT and surgery, with a 3-year OS of $95 \%$ vs. 79\%, respectively [3]. A meta-analysis of 40 SBRT studies (4850 patients) and 23 surgery studies (7071 patients) concluded that overall and disease free survival are similar after adjustments for operability and age [7].

Adjuvant chemotherapy after surgical resection has been shown to improve survival in certain patients with NSCLC [8]. This finding raises the question of whether adjuvant chemotherapy after lung SBRT could potentially benefit those at risk for metastatic failure. Predicting patients at increased risk for disease recurrence after SBRT could help guide treatment and lead to improved patient outcomes. Multiple studies have reported nodal and distant metastatic recurrence rates after lung SBRT for early stage NSCLC [2, 9-11]. The original Indiana University phase II study reported $8.6 \%$ nodal and $12.9 \%$ distant metastatic failure rates with a median follow up of 50 months [2]. RTOG 0236 reported a 3-year rate of disseminated failure of $22.1 \%$ and a 5 -year rate of $23.6 \%$ [1, 12]. The 5-year rate of regional failure was $10.9 \%$ [12]. The recently published SPACE trial (Stereotactic Precision And Conventional radiotherapy Evaluation) reported a distant failure rate of $24 \%$ and a regional failure rate (excluding local failures) of 7\% after SBRT [4]. In a large single-institution retrospective analysis of 676 patients treated with SBRT, with a median follow up of 32.9 months, the 2-year rates of regional and distant recurrences were $7.8 \%$ and $14.7 \%$, respectively. At 5 years, the rates were $12.7 \%$ and $19.9 \%$, respectively [10]. A review of 16 articles on SBRT in early stage NSCLC reported that the cumulative incidence of regional recurrences after SBRT varied from 0 to $28.6 \%$, with a mean incidence of $9.6 \%$ [11]. RTOG 0236 reported a 3-year distant metastatic recurrence rate of $5.9 \%$ for squamous cell carcinomas vs. $30.7 \%$ for non-squamous histologies, and 5-year rates of $5.9 \%$ and $31.6 \%$; though not statistically significant $[1,12]$. Moreover, the 5-year distant metastatic rates were $18 . \%$ for $\mathrm{T} 1$ tumors and $45.5 \%$ for $\mathrm{T} 2$ tumors [12].

Only a few studies have explored associations between clinical factors and the risk of nodal or distant metastatic failure after SBRT. A small study of 81 patients identified tumor size $(<2.5 \mathrm{vs.}$ $\geq 2.5 \mathrm{~cm}$ ), location (central vs. peripheral), stage (IA vs. IB), and use of certain drugs (metformin, statin, ACE inhibitor) as risk factors for distant metastasis; the final model achieved a 0.80 receiver operating characteristic (ROC) area under the curve (AUC) [13]. A similar effort using a larger cohort of 729 patients identified age, tumor size, FDG avidity, and presence of synchronous primaries as potential predictors of distant metastatic disease [14]. A nomogram built from these variables and several additional factors (body mass index (BMI), Charlson Comorbidity Index, medical operability) had a c-statistic of 0.606 [14]. Due to the limited 
available data and the clinical implications of metastatic failure for these patients, we sought to identify risk factors for metastatic recurrence after lung SBRT for early stage NSCLC.

\section{Materials and methods}

\section{Study population}

This study was an Institutional Review Board-approved retrospective review of outcomes after SBRT for NSCLC among patients treated between 2000 and 2016. All patients had provided informed consent for radiation therapy at the time of treatment. Patients were identified by medical billing codes or relevant billing information. Eligible patients were $\geq 18$ years of age with histologically or clinically diagnosed early stage NSCLC; synchronous lesions were included. A clinical diagnosis of NSCLC in patients who could not undergo or refused biopsy was based on radiographic suspicion, most often via review of serial chest CT scans in the setting of a multidisciplinary thoracic tumor board. Patients treated with SBRT were either medically inoperable or had refused surgery. Patients were excluded if they had evidence of systemic spread of lung cancer at the time of SBRT. Radiation planning and treatment details have been previously reported [15]. Prescriptions typically were to the $80 \%$ isodose line, and the prescription typically covered at least $95 \%$ of the PTV. More recently in the intensity-modulated radiation therapy setting, prescriptions were typically $95 \%$ of the PTV receiving $100 \%$ of the prescription and $99 \%$ of the internal target volume receiving at least $110 \%$ of the prescription. The most common dose fractionations are included in Table 1.

\section{Data collection}

The date of diagnosis was defined as the date of tumor sampling for those with a histologic diagnosis or the date of imaging prompting additional work up for those with a clinical diagnosis. The date of last follow-up was defined as the date the patient last visited with a radiation oncologist, medical oncologist, surgical oncologist, or pulmonologist. American Joint Committee on Cancer 7th edition (AJCC 7e) staging guidelines were used for all cases. Followup was done at the discretion of the treating physician. Follow-up imaging most often was by CT or positron emission tomography-CT (PET-CT). Recurrences were diagnosed by review of the patient's serial imaging and the clinical judgement of treating physicians. Distant sites were defined per AJCC 7e staging, with two exceptions: synchronous tumors were staged independently, and metachronous lung parenchymal lesions were staged as new primary lung cancers. Thus, limited contralateral lung parenchymal failures were considered a lung parenchymal failure and not a distant metastatic failure. Of note, only one patient in this cohort had three lung parenchymal failures over the course of seven years following SBRT before nodal or distant metastases were detected. All of the failures were managed with SBRT (one photon and two proton courses; proton courses not included in this analysis). Tumors were considered to be in a central location if the tumor's planning target volume (PTV) was located within $2 \mathrm{~cm}$ of the proximal tracheobronchial tree [16].

\section{Statistical analysis}


OS was calculated using the Kaplan-Meier method from the date of diagnosis until death or last follow-up. Time to metastases was defined as time from date of diagnosis to first nodal or distant metastasis, whichever occurred first, and censored at death or last follow-up. Age, smoking status (current, former, never, unknown), gross tumor volume (GTV), radiation prescription dose (biologically effective dose using a tumor alpha/beta $=10\left[\mathrm{BED}_{10}\right]$ ), and minimum, maximum, and mean dose to GTV (all in $\mathrm{BED}_{10}$ ) were considered continuous variables. Sex, lobe involved, location (central vs. peripheral), previous history of NSCLC, T stage, histology (adenocarcinoma, squamous cell carcinoma, NSCLC not otherwise specified, or no pathology), and parenchymal lung failure (local failure, same lobe non-local failure, other ipsilateral lobe failure, and contralateral lung failure) were considered categorical variables. Univariate analyses were used to determine the association between the aforementioned variables and time to metastatic failure. Significant variables on univariate analyses were then entered into the multivariate analyses. In addition, borderline significant radiation dose variables were also evaluated in separate multivariate analyses. A metastasis risk score linear model was built using beta coefficients from the multivariate Cox proportional hazards model (Table 3, model 1 using prescription dose instead of minimum, mean, or maximum dose because of ease of use and the ability to use the model before radiation planning is complete) as weighting factors for variables significant on multivariate analysis. Cutoffs were manually explored for risk score groupings and cutoffs -0.1 and -0.8 were used for further analysis as they were associated with the biggest differences in metastatic failure outcomes (see Figure 1). The performance of the metastasis risk score was evaluated by determining the $\mathrm{C}$-index using the Bioconductor/R rms (version 5.1-2) package by validation of the model using bootstrap (for details see table S2).

\section{Results}

From 2000 to 2016, 363 patients with 406 lesions met eligibility criteria and were treated with SBRT. Table 1 summarizes patient demographics and baseline characteristics. The majority of patients $(n=199,54.8 \%)$ were women, and median age was 73.2 years (range $44.1-93.8)$. The most common histologies were adenocarcinoma (40.8\%), squamous cell carcinoma $(32.2 \%)$, and NSCLC not otherwise specified $(17.4 \%) ; 9.6 \%$ were diagnosed clinically without pathologic confirmation. Overall, 117 (28.8\%) lesions were treated in the setting of presumed previously cured NSCLC. Patients were treated to a median $\mathrm{BED}_{10}$ of 105.6 Gy (range $72-244.8 \mathrm{~Gy}$ ). All patients had early-stage disease: $151 \mathrm{had}$ T1a (41.6\%), $120 \mathrm{~T} 1 \mathrm{~b}(33.1 \%)$, and $92 \mathrm{~T} 2 \mathrm{a}(25.3 \%)$ tumors. At least $93.5 \%$ of lesions were PET-staged. Only $6 \%$ of patients underwent invasive mediastinal staging, mostly by EBUS, at the treating physician's discretion. Median follow-up was 5.8 years.

Most patients had durable disease control; 252/406 lesions (62.1\%) and 224/363 patients (61.7\%) never recurred after SBRT. Nodal and/or distant failure occurred in 111/406 (27.3\%) total lesions, with a median time to first metastatic failure of 465 days (56 - 3784 days). The pattern of failure is as follows: 50 lesions had solely distant failure, 41 lesions had solely nodal failure, and 20 lesions failed in both sites. The remaining 43 lesions had either local or other parenchymal sites of failure, without nodal or distant failure.

Seventy of 406 (17.2\%) lesions failed distantly, with a median time to distant failure of 375 days (range 56 - 2704). The 1-, 2-yr, and 3-yr distant metastases-free survival rates were $94.6 \%$, $84.4 \%$, and $77.7 \%$, respectively. Median OS for patients with distant failure was 20 months 
(range 3.8 - 90.9 months). Nodal failure occurred in 61/406 (15.0\%) lesions, with a median time to nodal failure of 525 days (range 118 - 3784). Rates of 1-, 2-, and 3-year nodal metastases-free survival were $96.2 \%, 89.2 \%$, and $76.7 \%$, respectively. Median OS for patients with nodal failure was 25.5 months (range $5.2-131.7$ ). Of the 20 lesions $(4.9 \%)$ that had both distant and nodal failure, 9 were detected simultaneously, and 11 were detected at different times: 8 in nodes first and 3 distantly first.

GTV volume ( $p<0.005)$, GTV minimum dose $(p<0.05)$, and GTV mean dose $(p<0.05)$ were significantly associated with the development of metastases on univariate analysis. Radiation prescription dose, GTV maximum dose, histology, T stage, tumor centrality (figure S1), previous history of NSCLC (figure S2), or lung parenchymal failure after SBRT were not associated with the development of metastases ( $p>0.05$ ). Risk factors for individual failures are summarized in Table 2.

On multivariate Cox proportional hazards modeling (Table 3, Table S1), GTV mean dose and GTV volume remained significantly associated with time to metastasis ( $\mathrm{p}=0.042$ and $\mathrm{HR}=0.99$ per Gy and $\mathrm{p}<0.001$ and $\mathrm{HR}=1.02$ per $\mathrm{mL}$, respectively). Similarly, GTV volume and radiation prescription dose were significantly associated with time to metastasis on multivariate analysis ( $\mathrm{p}<0.001$, and $\mathrm{p}=0.044$, respectively), as were GTV maximum dose and GTV volume ( $\mathrm{p}=0.04$ and 0.0003 , respectively). Based on these findings, a metastasis risk score model using GTV (in $\mathrm{mL}$ ) and radiation prescription dose (in Gy) was built (see Table S2):

$\left[\right.$ Risk score $=(0.01611 \times$ GTV $)-\left(0.00525 \times\right.$ prescription dose $\left.\left.\left(\mathrm{BED}_{10}\right)\right)\right]$.

The distribution of risk scores in our cohort is shown in Figure 1. Consistent with its design, the risk score was significantly associated with time to metastatic failure $(H R=2.7, p<0.001)$. To illustrate the impact of the risk score on metastatic failure, two risk score cutoffs $(-0.8$ and -0.1$)$ separating the cohort into low- $(<-0.8)$, medium- $(-0.8$ to -0.1$)$, and high-risk $(>-0.1)$ groups were examined (figure 2 and S2). The risk score identified significant differences in time to metastases between 61 low risk, 264 medium risk, and 38 high risk patients ( $\mathrm{p}<0.001)$. The 1-, 2-, and 3year metastasis-free survival rates for the low-, medium-, and high-risk groups were $96.0 \%$, $86.7 \%$, and $81.1 \% ; 93.8 \%, 81.3 \%$, and $63.8 \%$; and $81.5 \%, 48.8 \%$, and $38.0 \%$, respectively (p < 0.0001 , table 4). Respective median OS times for the low-, medium-, and high-risk groups were 28.2 (range $2.4-143.9$ ), 19.9 (range $0.3-178.1$ ), and 15.5 months (range 1.4-69.3). The 1-yr, 2-yr, and 3-yr OS rates for the low-, medium-, and high-risk groups were 96.0\%, 86.7\%, and $83.9 \%$; $95.2 \%, 86.3 \%$, and $78.4 \%$; and $87.9 \%, 64.5 \%$, and $57.3 \%$, respectively.

\section{Discussion}

This study represents one of the first and largest studies dedicated to developing a risk score for either nodal or distant metastatic failure after treatment with SBRT for early stage NSCLC.

Distant metastasis rates in our cohort (17\%) were similar to most published series; however, the observed crude nodal failure rate $(15 \%)$ as well as nodal metastasis-free survival in our cohort was slightly higher than previous published series $[2,4,10]$.Together, a crude metastatic failure rate of $27 \%$ provided sufficient events $(n=111)$ to reasonably conduct the presented study. 
We identified radiation dose and GTV volume to be independently associated with metastatic failure. Our data suggests that lower prescription dose was associated with increased metastatic failure independent of GTV volume. To our knowledge, this is the first study to demonstrate the combined importance of GTV volume and radiation dose for metastatic failure risk. Other studies have also reported on associations between tumor size and the development of metastases after SBRT. In a phase II study by Baumann et al., T2 lesions were associated with significantly increased regional and distant recurrences at 3 years compared to T1 lesions [17]. RTOG 0236 reported similar findings [12]. Allibhai et al. prospectively analyzed 185 medically inoperable patients with T1-2N0M0 NSCLC who received SBRT. With a median follow up of 15.2 months, they found that larger GTV volume was a predictor for worse distant relapse-free survival [18]. Other studies similarly have shown that larger tumor size is associated with the development of distant metastasis $[13,14]$. However, tumor size was reported to not be associated with nodal failure after SBRT in a single institutional study [19]. Studies evaluating the presence of occult nodal metastases using invasive staging in patients with a PET-negative mediastinum suggest that larger tumor size and central tumor location are associated with nodal metastasis $[20,21]$. The details of a possible association between tumor size and the development of nodal metastases after SBRT remain to be determined. Radiation dose has been previously reported to be associated with a reduced nodal and distant metastasis failure rate. In a 91-patient study, prescription dose, but not GTV or PTV, was the only factor associated with combined nodal or distant metastatic failure risk [22].

Strengths of our study include the large number of events and long follow up. Limitations inherent in retrospective analyses also apply to our study. Moreover, our study included patients who were treated for presumed second primary NSCLC, and it is possible that some metastases were actually from a previously treated NSCLC. Of note, having a history of NSCLC that was previously treated and thought to be cured was not associated with the development of metastases in our cohort. Also, the availability and use of EBUS evolved over time, and only a small percentage of patients in this cohort underwent invasive mediastinal staging prior to SBRT. This difference may have resulted in selection bias, as it is possible that patients with larger central lesions, who are traditionally thought to be at higher risk for occult nodal metastasis [21], were more likely to undergo EBUS. This difference might also explain why centrality was not associated with metastasis in the studied cohort. We aim to increase the use of EBUS staging for our patients in the future. It remains unclear why GTV volume was found to be a significant predictor of metastatic failure, but $\mathrm{T}$ stage was not. While the combination of nodal and distant metastasis for this analysis increased the number of events, certain tumor, patient, or treatment factors associated with the risk of nodal vs. distant failure may differ, and our model was not able to account for these potential differences. Furthermore, it remains to be seen how this model performs on other lung SBRT cohorts with a different mix of patients, tumors, and treatments. Moreover, the prescription doses evaluated in this study represent only a subset of accepted and commonly used prescription doses. Thus, it remains unclear if our results translate to prescription doses other than the ones evaluated here. Clearly, independent validation is needed to determine the predictive ability of the identified signature.

In conclusion, we identified tumor volume and radiation dose to be associated with the development of metastases after SBRT for early stage NSCLC. Identification of patients at high 
risk for the development of metastases may inform future studies evaluating treatment intensification strategies (e.g., adjuvant chemotherapy or immunotherapy) for such patients.

\section{Clinical Practice Points}

Unfortunately, many early stage NSCLC (ES-NSCLC) patients develop metastasis even after successful local treatment with stereotactic body radiation therapy (SBRT), suggesting the presence of occult metastases at initial diagnosis. Upfront identification of patients at highest risk for metastasis would allow to select patients for additional workup to minimize the risk of harboring undetected metastases. We now aimed to not only identify factors predicting metastatic failure after lung SBRT for ES-NSCLC patients but also build a nomogram that can be used as a clinical decision tool for assessing patients that may benefit from undergoing more invasive staging procedures such as endobronchial ultrasound (EBUS) for mediastinal staging. We were able to identify factors associated with a higher risk of nodal and/or distant failure and build such a nomogram. Using this tool patients can be classified according to tumor size and radiation dose into low, intermediate, and high risk for metastases. The nomogram predicted metastasis free survival rates at 3 -years are $81.1 \%, 63.8 \%$, and $38 \%$ for the respective risk groups. We propose that i) patients at high risk for metastatic failure may benefit from EBUS if there are no strict contraindications, and ii) patients with low and intermediate risk should be counseled on the risks and benefits of additional staging.

\section{Acknowledgements}

The data analysis was performed by the Collaborative Core for Cancer Bioinformatics shared by IU Simon Cancer Center (Grant P30CA082709) and Purdue University Center for Cancer Research (Grant P30CA023168) with financial support from Walther Cancer Foundation. 


\section{$\underline{\text { References }}$}

1. Timmerman, R., et al., Stereotactic body radiation therapy for inoperable early stage lung cancer. JAMA, 2010. 303(11): p. 1070-6.

2. Fakiris, A.J., et al., Stereotactic body radiation therapy for early-stage non-small-cell lung carcinoma: four-year results of a prospective phase II study. Int J Radiat Oncol Biol Phys, 2009. 75(3): p. 677-82.

3. Chang, J.Y., et al., Stereotactic ablative radiotherapy versus lobectomy for operable stage I non-small-cell lung cancer: a pooled analysis of two randomised trials. Lancet Oncol, 2015. 16(6): p. 630-7.

4. Nyman, J., et al., SPACE - A randomized study of SBRT vs conventional fractionated radiotherapy in medically inoperable stage I NSCLC. Radiother Oncol, 2016. 121(1): p. $1-8$.

5. Videtic, G.M., et al., A Randomized Phase 2 Study Comparing 2 Stereotactic Body Radiation Therapy Schedules for Medically Inoperable Patients With Stage I Peripheral Non-Small Cell Lung Cancer: NRG Oncology RTOG 0915 (NCCTG N0927). Int J Radiat Oncol Biol Phys, 2015. 93(4): p. 757-64.

6. Bezjak, A., et al., Primary Study Endpoint Analysis for NRG Oncology/RTOG 0813 Trial of Stereotactic Body Radiation Therapy (SBRT) for Centrally Located Non-Small Cell Lung Cancer (NSCLC). International Journal of Radiation Oncology • Biology • Physics. 94(1): p. 5-6.

7. Zheng, X., et al., Survival outcome after stereotactic body radiation therapy and surgery for stage I non-small cell lung cancer: a meta-analysis. Int J Radiat Oncol Biol Phys, 2014. 90(3): p. 603-11.

8. $\quad$ Strauss, G.M., et al., Adjuvant paclitaxel plus carboplatin compared with observation in stage IB non-small-cell lung cancer: CALGB 9633 with the Cancer and Leukemia Group $B$, Radiation Therapy Oncology Group, and North Central Cancer Treatment Group Study Groups. J Clin Oncol, 2008. 26(31): p. 5043-51.

9. Giuliani, M.E., et al., Predictors and Patterns of Regional Recurrence Following Lung SBRT: A Report From the Elekta Lung Research Group. Clin Lung Cancer, 2017. 18(2): p. $162-168$.

10. Senthi, S., et al., Patterns of disease recurrence after stereotactic ablative radiotherapy for early stage non-small-cell lung cancer: a retrospective analysis. Lancet Oncol, 2012. 13(8): p. 802-9.

11. Wink, K.C.J., et al., Nodal recurrence after stereotactic body radiotherapy for early stage non-small cell lung cancer: Incidence and proposed risk factors. Cancer Treat Rev, 2017. 56: p. 8-15.

12. Timmerman, R.D., et al., Long-term Results of Stereotactic Body Radiation Therapy in Medically Inoperable Stage I Non-Small Cell Lung Cancer. JAMA Oncol, 2018.

13. Zhou, Z., et al., Predicting distant failure in early stage NSCLC treated with SBRT using clinical parameters. Radiother Oncol, 2016. 119(3): p. 501-4.

14. Oh, S., et al., A Novel Nomogram for Predicting Distant Metastases After Lung Stereotactic Body Radiation Therapy for Early-Stage Lung Cancer. International Journal of Radiation Oncology • Biology • Physics. 93(3): p. S103.

15. Shiue, K., et al., Histology, tumor volume, and radiation dose predict outcomes in nonsmall cell lung cancer patients after stereotactic ablative radiotherapy Journal of Thoracic Oncology, 2018. 
16. Timmerman, R., et al., Excessive toxicity when treating central tumors in a phase II study of stereotactic body radiation therapy for medically inoperable early-stage lung cancer. $\mathrm{J}$ Clin Oncol, 2006. 24(30): p. 4833-9.

17. Baumann, P., et al., Outcome in a prospective phase II trial of medically inoperable stage I non-small-cell lung cancer patients treated with stereotactic body radiotherapy. J Clin Oncol, 2009. 27(20): p. 3290-6.

18. Allibhai, Z., et al., The impact of tumor size on outcomes after stereotactic body radiation therapy for medically inoperable early-stage non-small cell lung cancer. Int J Radiat Oncol Biol Phys, 2013. 87(5): p. 1064-70.

19. Marwaha, G., et al., Lung stereotactic body radiation therapy: regional nodal failure is not predicted by tumor size. J Thorac Oncol, 2014. 9(11): p. 1693-7.

20. Gao, S.J., et al., Indications for invasive mediastinal staging in patients with early nonsmall cell lung cancer staged with PET-CT. Lung Cancer, 2017. 109: p. 36-41.

21. Cerra-Franco, A., K. Diab, and T. Lautenschlaeger, Undetected lymph node metastases in presumed early stage NSCLC SABR patients. Expert Rev Anticancer Ther, 2016. 16(8): p. 869-75.

22. Bradley, J.D., et al., Stereotactic body radiation therapy for early-stage non-small-cell lung cancer: the pattern of failure is distant. Int J Radiat Oncol Biol Phys, 2010. 77(4): p. 1146-50. 


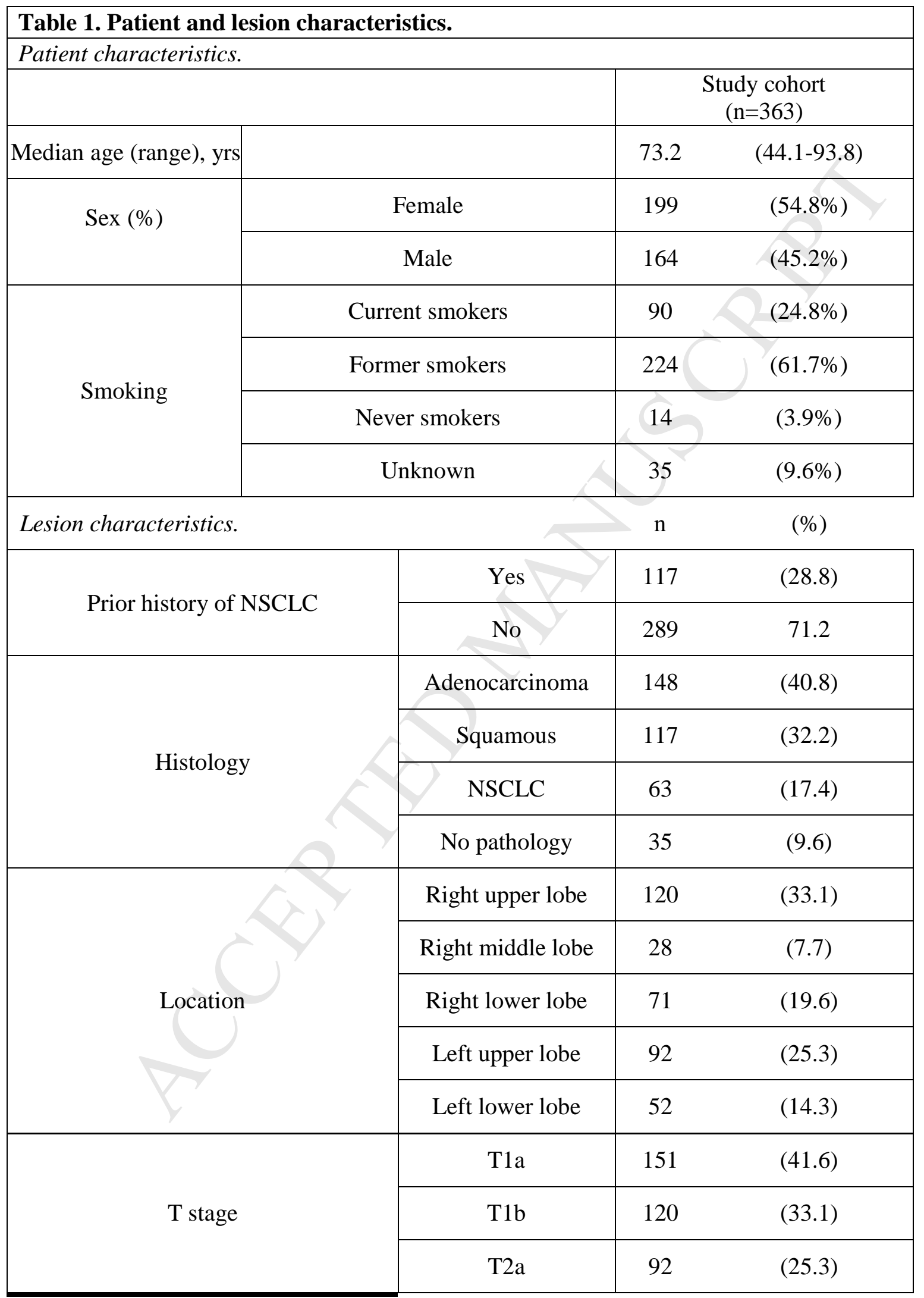




\begin{tabular}{|c|c|c|c|}
\hline \multirow{5}{*}{ Dose fractionations (BED10) } & 12 Gy x 4 (105.6 Gy) & 136 & $33.5 \%$ \\
\hline & 20 Gy x 3 (180 Gy) & 74 & $18.2 \%$ \\
\hline & 18 Gy x 3 (151.2 Gy) & 73 & $18.0 \%$ \\
\hline & 10 Gy x 5 (100 Gy) & 59 & $14.5 \%$ \\
\hline & Other & 64 & $15.8 \%$ \\
\hline Rx Dose* (range) & & $135.6 \mathrm{~Gy}$ & $(72.0-244.8)$ \\
\hline GTV minimum dose* (range) & & $152.1 \mathrm{~Gy}$ & $(77.6-292.3)$ \\
\hline GTV maximum dose* (range) & & 195.4 Gy & $(100.8-374.0)$ \\
\hline GTV mean dose* (range) & & $180.3 \mathrm{~Gy}$ & $(94.3-339.5)$ \\
\hline GTV (range) & & $14.2 \mathrm{~mL}$ & $(0.5-156.7)$ \\
\hline $\begin{array}{r}\text { Local failure (range) } \\
\text { Days to failure }\end{array}$ & 31 failures & $1027.0^{\dagger}$ & $(168-2812)$ \\
\hline $\begin{array}{r}\text { Same lobe non-local failure (range) } \\
\text { Days to failure }\end{array}$ & 26 failures & $778.7^{\dagger}$ & $(26-164)$ \\
\hline $\begin{array}{r}\text { Other ipsilateral lobe failure (range) } \\
\text { Days to failure }\end{array}$ & 33 failures & $840.0^{\dagger}$ & $(92-2142)$ \\
\hline $\begin{array}{r}\text { Contralateral lung failure (range) } \\
\text { Days to failure }\end{array}$ & 45 failures & $956.0^{\dagger}$ & $(118-3784)$ \\
\hline
\end{tabular}

*Doses expressed in $\mathrm{BED}_{10}$.

${ }^{\dagger}$ Mean number of days to failure

NSCLC: non-small cell lung cancer; Rx: prescription; GTV: gross tumor volume (in mL); Gy: Gray; mL: milliliters; d: days; yr: years. 
Table 2. Univariate analysis for metastatic failure

\begin{tabular}{|l|c|}
\hline Variable & p-value \\
\hline Sex & 0.36 \\
\hline Age & 0.40 \\
\hline Current smoker & 0.46 \\
\hline Former smoker & 0.44 \\
\hline Lobe location & 0.94 \\
\hline T stage & 0.49 \\
\hline Histology & 0.47 \\
\hline Central vs. peripheral & 0.55 \\
\hline Previous NSCLC & 0.84 \\
\hline Rx dose & $\mathbf{0 . 0 5 9}$ \\
\hline GTV minimum dose & $\mathbf{0 . 0 2 7}$ \\
\hline GTV maximum dose & $\mathbf{0 . 0 5 5}$ \\
\hline GTV mean dose & $\mathbf{0 . 0 4 4}$ \\
\hline GTV volume & $\mathbf{0 . 0 0 4}$ \\
\hline Local failure & 0.38 \\
\hline Same lobe non-local failure & 0.51 \\
\hline Other ipsilateral lobe failure & 0.37 \\
\hline Contralateral lung failure & 0.11 \\
\hline NSCLC: non-smal cell lung
\end{tabular}

NSCLC: non-small cell lung cancer; Rx: prescription; GTV: gross tumor volume (in $\mathrm{mL}$ ). 
Table 3. Multivariate Analysis for metastatic failure

\begin{tabular}{|c|l|c|c|c|}
\hline Model \# & Variable & p-value & $95 \%$ CI & HR \\
\hline \multirow{3}{*}{1} & Rx dose & 0.044 & $\begin{array}{c}0.98957- \\
0.99997\end{array}$ & 0.99477 \\
\cline { 2 - 5 } & GTV volume & 0.0003 & $\begin{array}{c}1.0073- \\
1.02518\end{array}$ & 1.01624 \\
\hline
\end{tabular}

Rx: prescription; GTV: gross tumor volume (in $\mathrm{mL}$ ). 
Table 4. Metastases-free survival

\begin{tabular}{|c|c|c|c|}
\hline & Low risk & Medium risk & High risk \\
\hline 1-yr MFS & $96.0 \%$ & $93.8 \%$ & $81.5 \%$ \\
\hline 2-yr MFS & $86.7 \%$ & $81.3 \%$ & $48.8 \%$ \\
\hline 3-yr MFS & $81.1 \%$ & $63.8 \%$ & $38.0 \%$ \\
\hline 1-yr NFS & $98.0 \%$ & $96.2 \%$ & $92.7 \%$ \\
\hline 2-yr NFS & $98.0 \%$ & $88.7 \%$ & $75.7 \%$ \\
\hline 3-yr NFS & $94.7 \%$ & $72.8 \%$ & $66.2 \%$ \\
\hline Median OS & 28.2 months & 19.9 months & 15.5 months \\
\hline
\end{tabular}

MFS: metastases-free survival (combined nodal and distant metastatic failure); NFS: nodalfailure-free survival OS: overall survival. 


\section{Figure Captions}

Figure 1. Distribution of risk scores.

Figure 2. Kaplan Meier curves for metastasis free survival according to risk score. 
Histogram of risk score

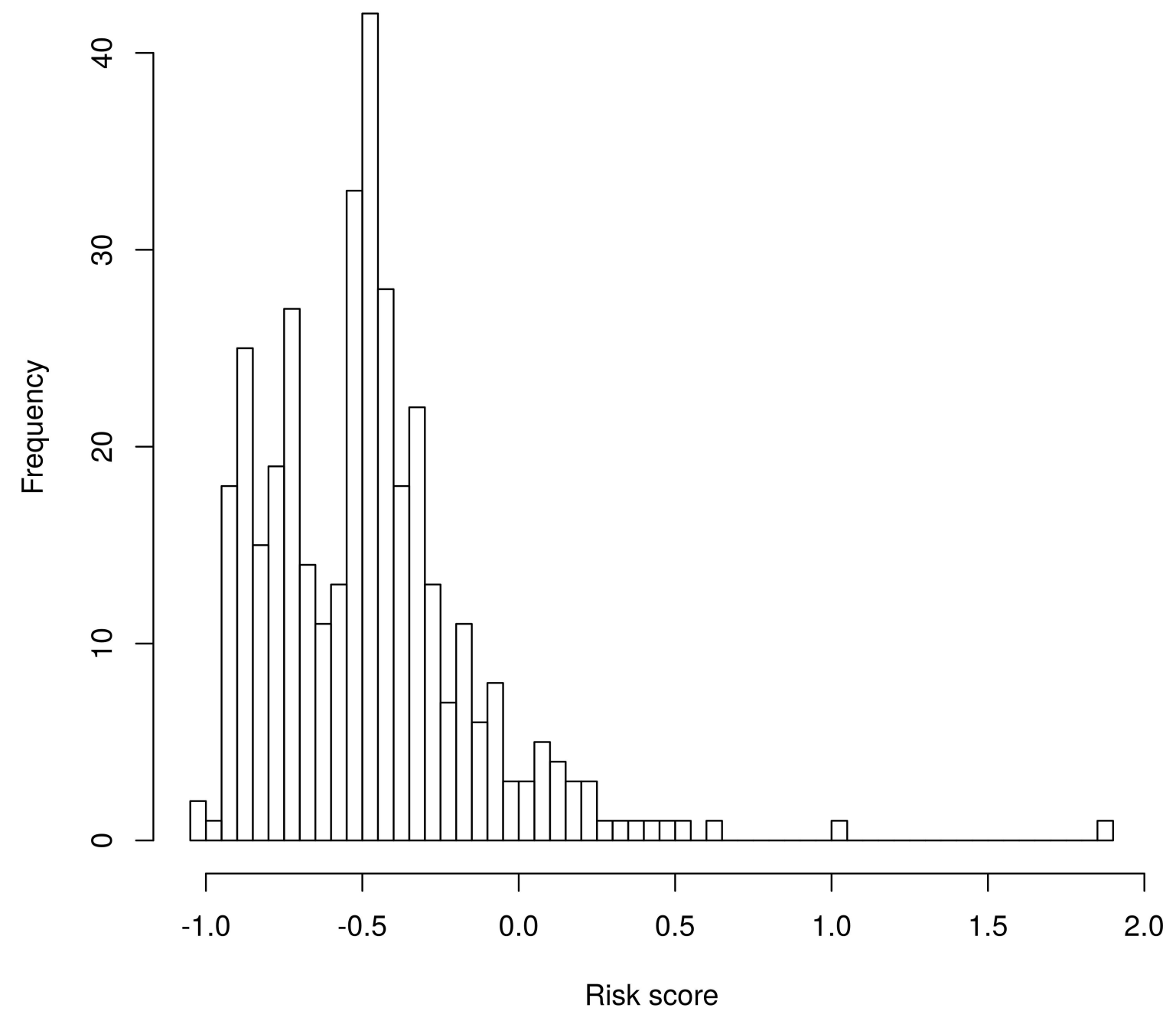




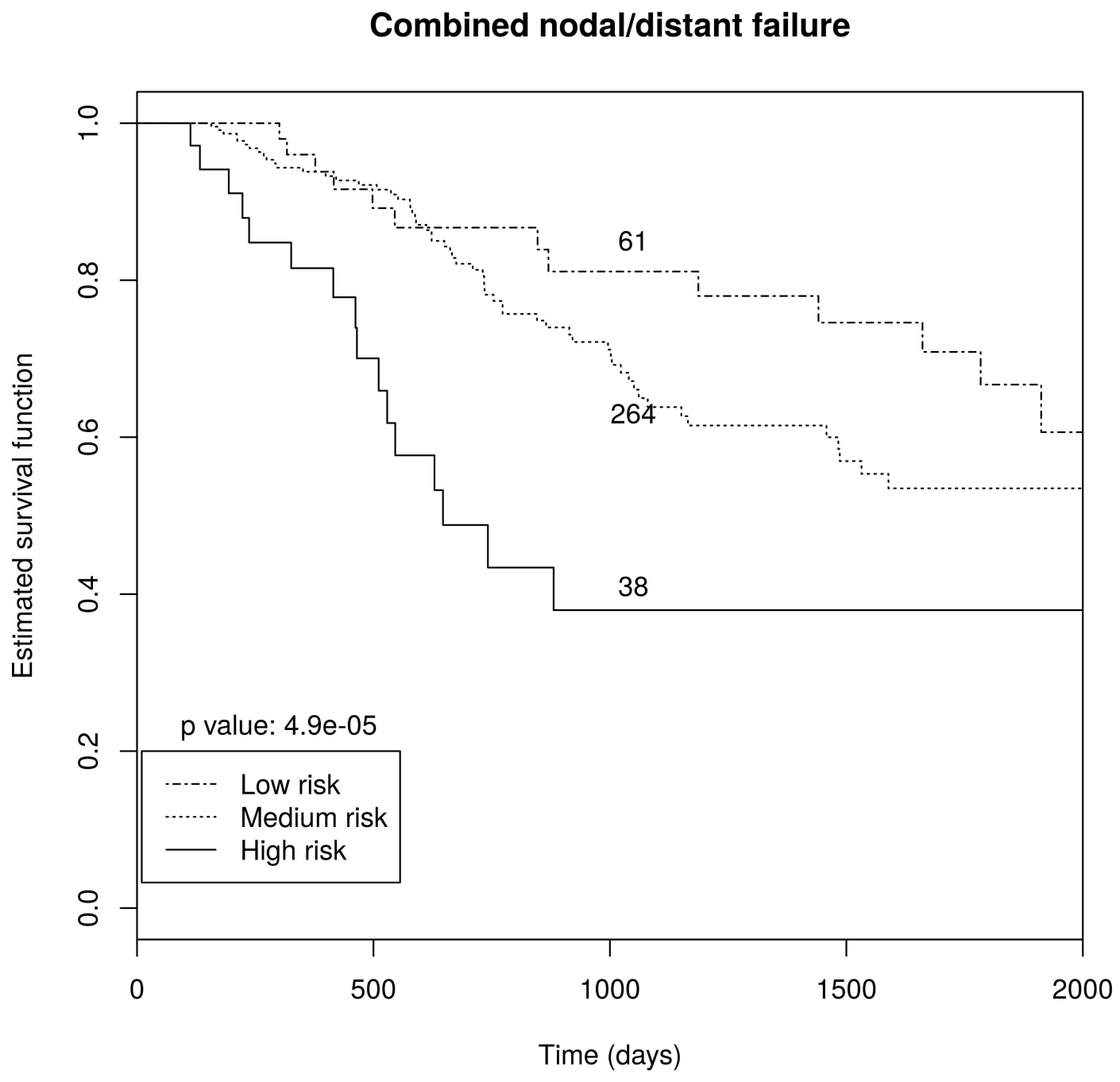

\begin{tabular}{|c|c|c|c|c|c|}
\hline \multirow{5}{*}{ Low risk } & Time (days) & \# at risk & MFS & lower $95 \% \mathrm{CI}$ & upper $95 \% \mathrm{CI}$ \\
\hline & 0 & 61 & & & \\
\hline & 365 & 46 & 0.96 & 0.907 & 1 \\
\hline & 730 & 32 & 0.867 & 0.773 & 0.973 \\
\hline & 1095 & 26 & 0.811 & 0.7 & 0.94 \\
\hline \multirow{5}{*}{ Medium risk } & Time (days) & \# at risk & MFS & lower $95 \% \mathrm{CI}$ & upper $95 \% \mathrm{CI}$ \\
\hline & 0 & 264 & & & \\
\hline & 365 & 175 & 0.938 & 0.906 & 0.971 \\
\hline & 730 & 103 & 0.813 & 0.755 & 0.875 \\
\hline & 1095 & 56 & 0.638 & 0.559 & 0.729 \\
\hline \multirow{5}{*}{ High risk } & Time (days) & \# at risk & MFS & lower $95 \% \mathrm{CI}$ & upper $95 \% \mathrm{CI}$ \\
\hline & 0 & 31 & & & \\
\hline & 365 & 24 & 0.815 & 0.692 & 0.961 \\
\hline & 730 & 9 & 0.488 & 0.328 & 0.727 \\
\hline & 1095 & 5 & 0.38 & 0.223 & 0.645 \\
\hline
\end{tabular}

CI, confidence interval; MFS, metastasis-free survival. 
Figure S1. Combined nodal and distant failure according to centrality.

\section{Combined nodal/distant failure}

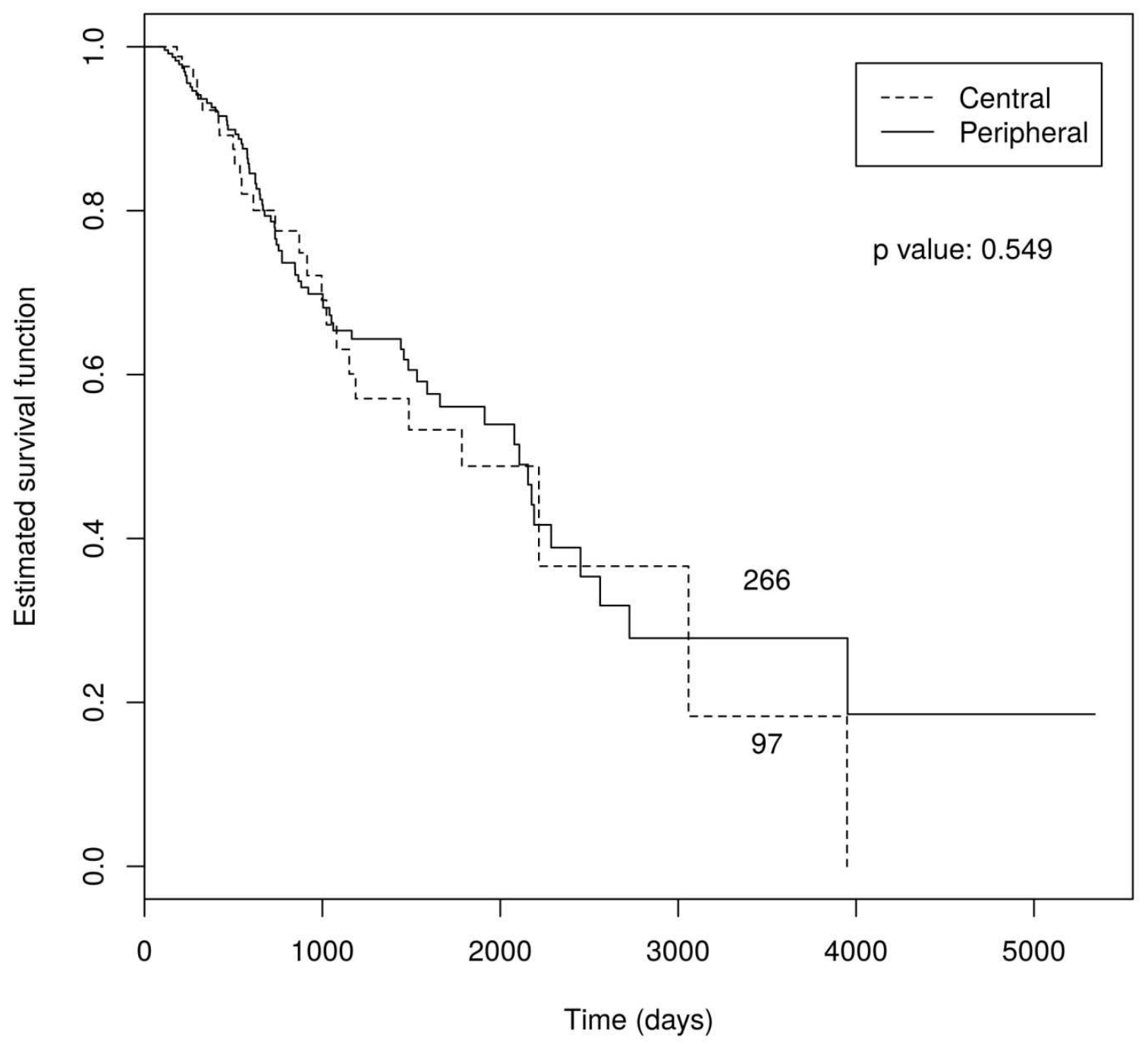


Figure S2. Risk of distant failure stratified by low-, medium-, and high risk in patients without a prior history of non-small cell lung cancer.

\section{Combined nodal/distant failure}

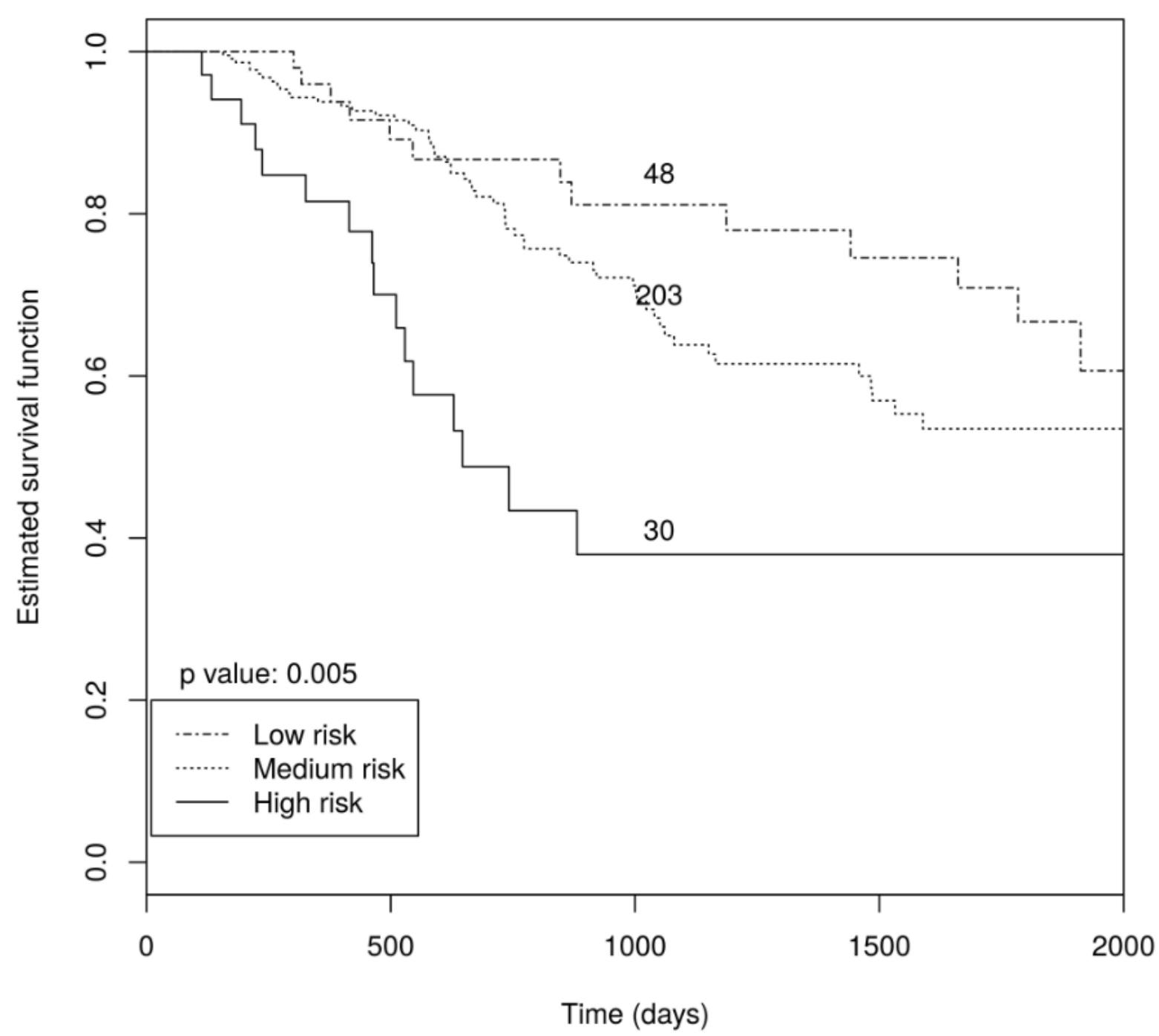


Table S1 Additional Multivariate Analysis for metastatic failure

\begin{tabular}{|c|l|c|c|c|}
\hline \multirow{2}{*}{2} & GTV minimum dose & 0.059 & $\begin{array}{c}0.99172- \\
1.00024\end{array}$ & 0.99598 \\
\cline { 2 - 5 } & GTV volume & 0.002 & $\begin{array}{r}1.0053- \\
1.02362\end{array}$ & 1.01446 \\
\hline \multirow{3}{*}{3} & GTV mean dose & 0.042 & $\begin{array}{c}0.99276- \\
0.99996\end{array}$ & 0.99630 \\
\cline { 2 - 5 } & GTV volume & 0.0005 & $\begin{array}{c}1.00686- \\
1.02478\end{array}$ & 1.01582 \\
\hline \multirow{3}{*}{4} & GTV maximum dose & 0.04 & $\begin{array}{c}0.99343- \\
0.99991\end{array}$ & 0.99667 \\
\cline { 2 - 5 } & GTV volume & 0.0003 & $\begin{array}{c}1.00734- \\
1.02522\end{array}$ & 1.01628 \\
\hline
\end{tabular}

Rx: prescription; GTV: gross tumor volume (in $\mathrm{mL}$ ).

Table S2 Prescription dose and GTV volume model

\begin{tabular}{lllllll}
\hline values & index.orig & training & test & optimism & index.corrected & n \\
\hline Dxy & 0.244937073 & 0.243806916 & 0.234935284 & 0.008871632 & 0.236065441 & 1000 \\
\hline R2 & 0.036866192 & 0.042419252 & 0.033556826 & 0.008862426 & 0.028003767 & 1000 \\
\hline Slope & 1.000000000 & 1.000000000 & 0.958265124 & 0.041734876 & 0.958265124 & 1000 \\
\hline D & 0.012242654 & 0.014324331 & 0.011033412 & 0.003290919 & 0.008951736 & 1000 \\
\hline U & -0.002107209 & -0.002124432 & 0.002471792 & -0.004596224 & 0.002489014 & 1000 \\
\hline Q & 0.014349864 & 0.016448763 & 0.008561620 & 0.007887142 & 0.006462721 & 1000 \\
\hline
\end{tabular}

Somers' Dxy is equal to 2* (C-index -0.5 ), and C-index is the concordance probability. $\mathrm{R}^{2}$ is goodness of fit. $\mathrm{D}$ is discrimination telling the difference in quality of the best constant predictor and the best calibrated predictor. $U$ is unreliability of the predictions, $Q$ is overall summary showing the difference in quality between the best constant predictor and the quality of the predictions when no calibration was made. The c-index value is 0.618 . 